Classification

Physics Abstracts

$42.65 \mathrm{~J}-32.80 \mathrm{P}$

\title{
Limitation of the Polarization by Radiation Trapping in a Helium Afterglow Electron Source
}

\author{
I. Brissaud and C. Jacquemin \\ Instıtut de Physique Nucléaire, 91406 Orsay Cedex, France
}

(Received 7 Aprll 1994, revised 23 September 1994, accepted 28 September 1994)

\begin{abstract}
A polarized electron source using an optically pumped helium afterglow was built at Orsay. Unfortunately the spin polarization decreases at high metastable densities. Calculations of the radiation trapping effects in a weak magnetic field are presented using the Anderson formalism. Comparison with experimental data leads to the conclusion that these trapping effects are one explanation of this polarization decrease. Effects of the main parameters are studied. Some deductions for a new desıgn can be made.
\end{abstract}

\section{Introduction}

A polarized electron source was built at Orsay [1] using an optically pumped helium afterglow. This source is adapted from the Rice University model designed by Walters et al. [2]. Metastable helium atoms in the $2^{3} \mathrm{~S}_{1}$ state are polarized by optical pumping to the $2^{3} \mathrm{P}_{0}$ state by polarized light. Electrons are freed through a chemi-ionization process such that the polarization of the metastables and of the electrons should be the same. Therefore $100 \%$ polarization is expected. However in the Orsay experiment [3] the electron polarization values vary from $80 \pm 7 \%$ for low helium pressure (i.e. low metastable density) to $55 \pm 5 \%$ for high pressure (i.e. high metastable density). A similar effect was also observed in a previous work [2]. A possible explanation for this depolarization phenomenon could be resonance radiation trapping, i.e. the reabsorption of unpolarized deexcitation photons in the gas. This effect is strongly dependent on the metastable densities in the media In this paper we present our analysis of this question. However, in order to explain the polarization loss at low helium pressure, where the radiation trapping is expected to be negligible, one also has to take into account intrinsic depolarization factors. These might be; i) the imperfect efficiency of the experimental apparatus: incomplete polarization of the laser light, beams slightly out of alignments, and ii) physical difficulties: metastable atoms in the singlet state $2^{1} \mathrm{~S}_{0}$ (estimated to be less than $5 \%$ ), polarization degradation in the chemi-ionization process, etc. Each of these factors may account for a polarization loss of a few percent. Thus perhaps $10 \%$ metastable depolarization could be accounted for by all these mechanisms. 


\section{Experimental Set-Up}

A high purity helium jet is ionized and excited through a Laval nozzle microwave discharge cavity (Fig. 1). Electrons and ions recombine at the wall of a $35 \mathrm{~cm}$ long, $10 \mathrm{~cm}$ diameter pyrex tube forming a mixture of ground state and metastable $2^{3} \mathrm{~S}_{1}$ helium atoms. By the action of a powerful Roots blower the bulk gas flow has an average speed of $1.2 \times 10^{4} \mathrm{~cm} . \mathrm{s}^{-1}$ in the pumping chamber. For a helium pressure of $5 \times 10^{-2}$ to $2 \times 10^{-1} \mathrm{mb}$ (approximately $10^{16}$ atoms $\mathrm{cm}^{-3}$ helium density) the metastable density is several times $10^{10} \mathrm{~cm}^{-3}$. The destruction of metastables at the wall yields an exponential rate of decay, down the tube, of the metastable density. The diffusion decay length is $\mathrm{dd}=\alpha \cdot R^{2} \cdot v \cdot P /(D P)$ where $R$ is the tube radius, $v$ the average gas speed, $P$ the helium pressure and $D$ the diffusion coefficient. In our case, between the molecular and the viscous flow case, $\alpha$ is close to 0.27 and $D P$ is $470 \mathrm{~cm}^{2}$ torr s s $^{-1}$ $[4,5]$. The measured and calculated values of dd are between 10 and $15 \mathrm{~cm}$

The horizontal earth magnetic field components are cancelled by two pairs of Helmholtz coils while a third pair produces a 2.5 gauss vertical field defining the quantization axis. The optical pumping is done by a circularly polarized $\sigma$ light beam parallel to the magnetic field and a linearly polarized $\pi$ light beam perpendicular to this field. These beams have approximately equal intensities and are provided by a multimode LNA laser through a beam splitter. Figure 2 shows the electric dipole transition at $1.083 \mu \mathrm{m}$ wavelength between the $2^{3} S_{1}$ and $2^{3} \mathrm{P}_{0}$ states. The pumping occurs in a $10 \mathrm{~cm}$ cubic chamber attached to the end of the pyrex tube [3]. The light beams have about the same diameter as the windows of the pumping chamber. The metastable densities are measured by the absorption of a $\lambda=1.083 \mu \mathrm{m}$ probe beam provided

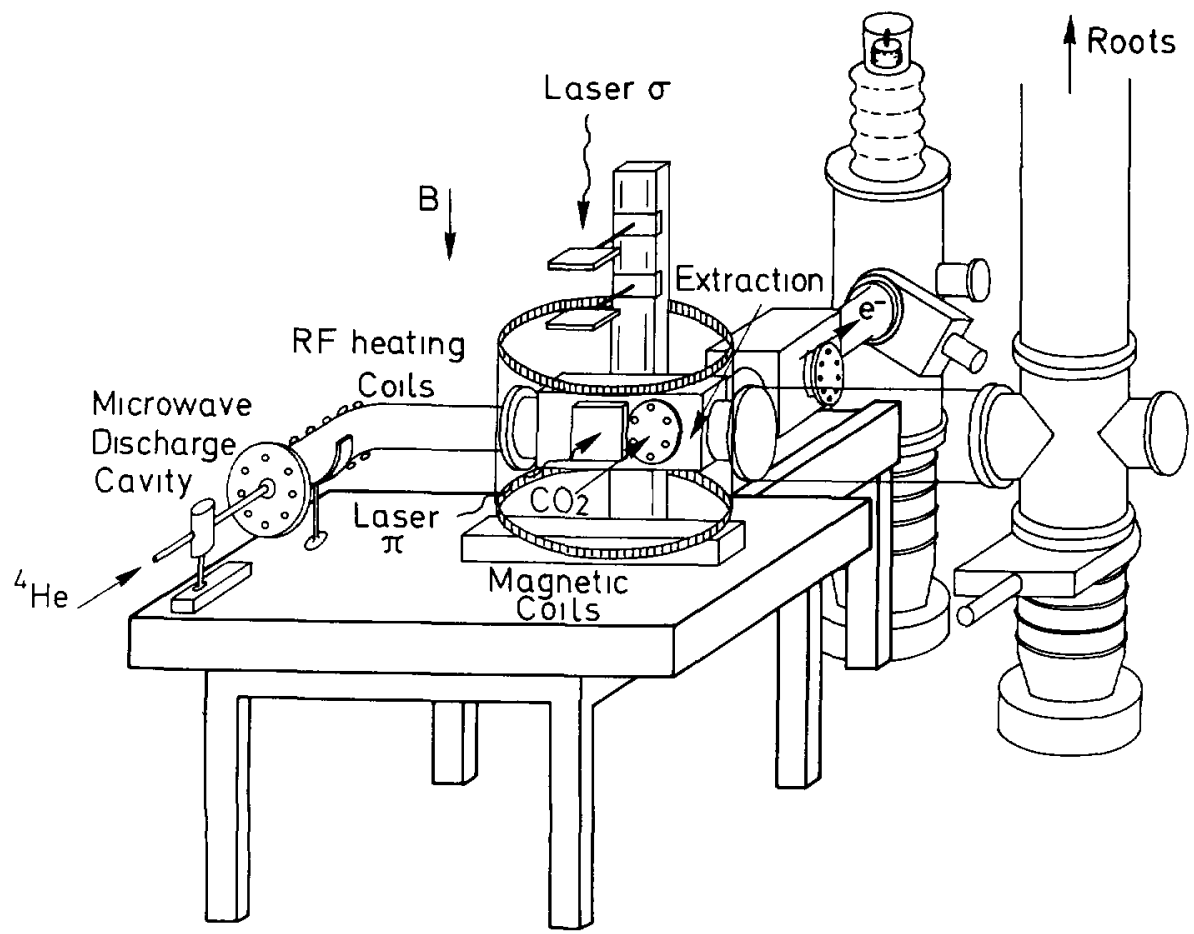

Fig. 1 - Experimental diagram of the Orsay source. 


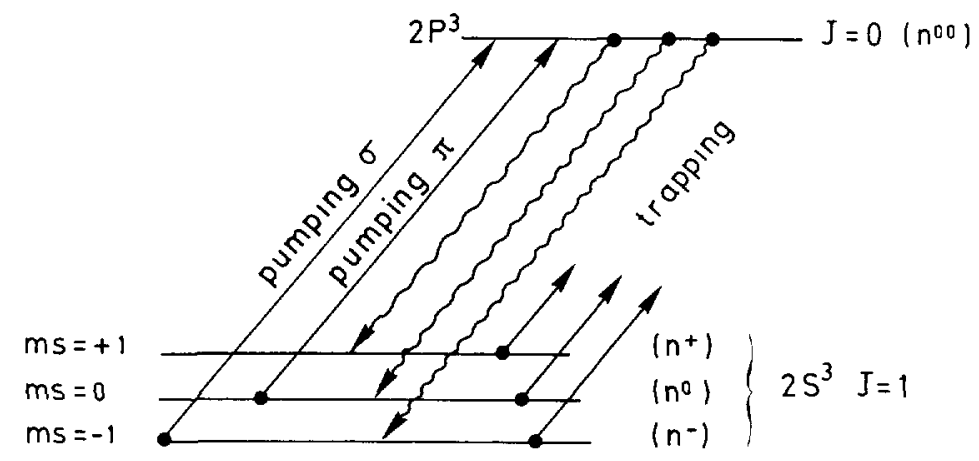

Fig. 2. - Energy levels of the helium metastable atoms. The $\sigma^{+}$and $\pi$ transitions used for optical pumping are shown. Spontaneous emissions from the $2^{3} \mathrm{P}_{0}$ level reabsorbed by the metastables are presented.

by a helium lamp or by a single mode, low power, diode pumped, LNA laser [6]. In this work the absorption is typically 10 to $20 \%$.

The Doppler width, $\Delta=\sqrt{2 k T \ell \mathrm{n} 2 / M}(\lambda, k, T, M$ are the pumping wavelength, the Boltzmann constant, the absolute temperature and the helium mass respectively) is very wide compared to the Zeeman splitting of the $2^{3} \mathrm{~S}_{1}$ levels in the very weak magnetic field (Landé $g$ factor $=2$ ). Therefore the radiation emitted by the transitions from the $2^{3} \mathrm{P}_{0}$ state to any one of the $2^{3} S_{1}$ substates can be reabsorbed by any one of the three magnetic substates of the $2^{3} S_{1}$ metastable atoms. This consideration explains the large probability of the trapping effect.

\section{Radiation Trapping Evaluation}

The densities of the metastable atoms in $m=+1,0,-1$ substates of the $2^{3} \mathrm{~S}_{1}$ triplet are $n^{+}(\overline{\mathrm{r}}, t), n^{0}(\overline{\mathrm{r}}, t), n^{-}(\overline{\mathrm{r}}, t)$ respectively while $n^{00}(\overline{\mathrm{r}}, t)$ represents the density for the $2^{3} \mathrm{P}_{0}$ state. At time $t$ and position $\bar{r}$ the total excited state density is thus $n=n^{+}+n^{0}+n^{-}+n^{00}$ (the $n^{2}(\overline{\mathrm{r}}, t)$ being systematically replaced below by $n^{2}$ for convenience). The four rate equations describing the densities in the various excited states are in the case of $\sigma^{+}$and $\pi$ pumping, without radiation reabsorption:

$$
\begin{aligned}
\frac{\mathrm{d} n^{-}}{\mathrm{d} t} & =-\frac{n^{-}}{\tau_{\mathrm{p}}}+n^{00}\left(\frac{1}{3 \tau}+\frac{1}{\tau_{\mathrm{p}}}\right)-\frac{n^{-}}{\tau_{\mathrm{r}}} \\
\frac{\mathrm{d} n^{0}}{\mathrm{~d} t} & =-\frac{n^{0}}{\tau_{\mathrm{p}^{\prime}}}+n^{00}\left(\frac{1}{3 \tau}+\frac{1}{\tau_{\mathrm{p}^{\prime}}}\right)-\frac{n^{0}}{\tau_{\mathrm{r}}} \\
\frac{\mathrm{d} n^{+}}{\mathrm{d} t} & =\frac{n^{00}}{3 \tau}-\frac{n^{+}}{\tau_{\mathrm{r}}} \\
\frac{\mathrm{d} n^{00}}{\mathrm{~d} t} & =\frac{n^{-}}{\tau_{\mathrm{p}}}+\frac{n^{0}}{\tau_{\mathrm{p}^{\prime}}}-n^{00}\left(\frac{1}{\tau}+\frac{1}{\tau_{\mathrm{p}}}+\frac{1}{\tau_{\mathrm{p}^{\prime}}}\right)
\end{aligned}
$$

These equations describe what is happening as a function of time in the reference frame of the gas as it enters the optical pumping region until it leaves, and $\tau_{\mathrm{r}}$ is the relaxation time. $\tau_{\mathrm{p}}$ and $\tau_{\mathrm{p}^{\prime}}$ are the pumping time constants of the $\sigma$ and $\pi$ light beams. These constants have 
the form $1 / \tau_{\mathrm{p}}=\frac{\sigma_{\sigma} \cdot I_{\sigma}}{h v}$ and $1 / \tau_{\mathbf{p}^{\prime}}=\frac{\sigma_{\pi} \cdot I_{\pi}}{h v}$ where $I_{\sigma}$ and $I_{\pi}$ are the intensities per unit area for the light source and $\nu$ is the frequency of this radiation. $\sigma_{\sigma}$ and $\sigma_{\pi}$ are the absorption cross sections for the circularly and linearly polarized radiation depending on the normalized Doppler lineshape

$$
F\left(v-v_{0}\right)=\left[\frac{M c^{2}}{2 \pi k T v_{0}^{2}}\right]^{1 / 2} \exp \left[-\left(\frac{v-v_{0}}{v_{0}}\right)^{2} \frac{M c^{2}}{2 k T}\right],
$$

with $v_{0}=c / \lambda_{0}, \lambda_{0}$ being the resonance wave length $1.083 \mu \mathrm{m}$.

A correction due to the laser width $\left(2.5 \times 10^{9} \mathrm{~s}^{-1}\right)$ is deduced following the procedure suggested by Mitchell and Zemanski [7]. This correction reduces the cross sections $\sigma_{\sigma}$ and $\sigma_{\pi}$ approximately by a factor 2 at $\lambda_{0}$. These cross sections are the state-to-state absorption cross section for the $2^{3} \mathrm{~S}_{1}\left(m_{\mathrm{s}}=-1\right) \rightarrow 2^{3} \mathrm{P}_{0}\left(m_{\mathrm{s}}=0\right)$ and $2^{3} \mathrm{~S}_{1}(m=0) \rightarrow 2^{3} \mathrm{P}_{0}(m=0)$ transitions respectively. $\sigma_{\sigma}$ and $\sigma_{\pi}$ can be calculated from the classical level-to-level $2^{3} \mathrm{~S}_{1} \rightarrow 2^{3} \mathrm{P}_{0}$ absorption cross section:

$$
\sigma_{\mathrm{ll}}=\frac{g_{\mathrm{f}}}{g_{\mathrm{l}}} \cdot \frac{\lambda^{2}}{8 \pi \tau} F\left(v-v_{0}\right)
$$

where $g_{\mathrm{f}}$ and $g_{1}$ are the final and initial state statistical weights These quantities are provided by means of the matrix elements of the components of the electric dipole operator $\mathrm{p}$ : $\left\langle\jmath^{\prime} m^{\prime}\left|p^{\delta}\right| \jmath m\right\rangle$ with $\delta=+1,0,-1[8]$. From these matrix elements one finds that all three transitions $2^{3} \mathrm{P}_{0} \rightarrow 2^{3} \mathrm{~S}_{1}$ have the same probability. Note that the level-to-level absorption cross sections (2) are for unpolarized light. The three state-to-state absorption cross sections are equal to $\frac{\lambda^{2}}{8 \pi \tau} F\left(v-v_{0}\right)$. In equations (1) the $n^{00} / \tau_{\mathrm{p}}$ and $n^{00} / \tau_{\mathrm{p}^{\prime}}$ terms correspond to the stimulated transitions and are very weak and negligible.

The relaxation time $\tau_{\mathbf{r}}$ is governed by the metastable diffusion to the walls [9]. It can be evaluated from classical diffusion theory [10] as $\tau_{\mathrm{r}}=\alpha \cdot R^{2} P /(D P)$. A complete discussion of the measurements and calculations of $\tau_{\mathrm{r}}$ has been achieved by Rice [4].

To take the radiation trapping effects into account, we use the procedure proposed by Anderson and coworkers [10]. We summarise this procedure as follows:

At point $\overline{\mathrm{r}}^{\prime}$, an atom in the $2^{3} \mathrm{P}_{0}$ state decays to any of the three $2^{3} \mathrm{~S}_{1}$ substates and the emitted radiation is absorbed at a point $\overline{\mathrm{r}}$ by a metastable in any of the three $2^{3} \mathrm{~S}_{1}$ substates. Then the trapping contribution [10] to the rate equations (1) is, for the example of the $n^{+}$ density:

$$
\begin{aligned}
& \frac{\mathrm{d} n^{+}(\overline{\mathrm{r}}, t)}{\mathrm{d} t}= \\
& -n^{00} \int \sum_{\imath} \mathrm{d}^{3} \overline{\mathrm{r}} \mathrm{d} v P_{\jmath}(\theta)\left\{\exp -\sum_{\imath} \sigma_{\jmath}^{2}(\theta)\left(n^{2}-n^{00}\right)(\overline{\mathrm{r}}-\overline{\mathrm{r}})\right\} \sigma_{\jmath}^{2}(\theta)\left(n^{+}-n^{00}\right),
\end{aligned}
$$

where $P_{3}(\theta)$ is the dipolar emission probability between the level $2^{3} \mathrm{P}$ and the $\jmath$ Zeeman substate of the $2^{3} \mathrm{~S}$ level. $\sigma_{\jmath}^{2}(\theta)$ is the absorption cross section of the radiation emitted by the deexcitation of $2^{3} \mathrm{P} \rightarrow 2^{3} \mathrm{~S}$ (in the Zeeman substate $m=\jmath$ ) by atoms in the Zeeman substate $m=\imath$. This radiation is polarized and its polarization is defined by the $\Delta m$ selection rule. In the quantities $\sigma_{\jmath}^{\imath}(\theta), \imath$ and $\jmath$ represent the $\Delta m$ values of the radiation emission $(\imath)$ and of the radiation absorption $(J)$ in the trapping process. The nine values $\sigma_{\jmath}^{2}(\theta)$ are given in Appendix. Here $\theta$ is the angle between the drection $\overline{\mathrm{r}}-\overline{\mathrm{r}}^{\prime}$ and the quantization axis. The exponential term shows the absorption of radiation between $\overline{\mathrm{r}}$ and $\overline{\mathrm{r}}^{\prime}$ while the last term is the absorption 
probability at the point $\overrightarrow{\mathbf{r}}$. Similar expressions for $n^{-}$and $n^{0}$ can be obtained. In addition, there is a fourth contribution to the rate equations. It is due to the $2^{3} \mathrm{P}$ state and can be written as $\frac{\mathrm{d} n^{00}}{\mathrm{~d} t}=-\sum_{\imath} \frac{\mathrm{d} n^{\imath}}{\mathrm{d} t}$.

These integro-differential equations are nonlinear and nonlocal. In order to simplify the numerical calculations of these equations the following assumptions are made:

i) the state populations $n^{+}, n^{0}, n^{-}, n^{00}$ are independent of $\overline{\mathbf{r}}$ inside the pumping chamber;

ii) the intensity of the laser light is uniform on the window of the chamber.

The first assumption, to be discussed later, allows an analytical integration over $\overline{\mathrm{r}}$. The integrations over frequency and angle are performed by using Hermitian-Gaussian approximation methods. We denote by $\chi_{2}$ the nodes and by $\omega_{2}$ the weighting factors of the Hermite integration method [11], and denote by $u_{j}$ the nodes and by $\omega_{\imath}$ the weighting factors of the Gauss integration method [11]. Also $\gamma_{2}=\frac{\lambda^{3}}{8 \pi \tau}\left[\frac{M}{2 \pi k T}\right]^{1 / 2} \mathrm{e}^{-\chi_{\imath}^{2}}$. This yields the following expression in the example of $n^{-}$:

$$
\frac{\mathrm{d} n^{-}}{\mathrm{d} t}=-\frac{n^{00}}{2 \sqrt{\pi} \tau}\left(n^{-}-n^{00}\right) \sum_{\imath} \omega_{2} \sum_{\imath} \omega_{1 \jmath} F_{1}\left(u_{\jmath}\right)+\omega_{3 \jmath} F_{3}\left(u_{\jmath}\right)+\omega_{5 \jmath} F_{5}\left(u_{\jmath}\right)
$$

with

$$
\begin{aligned}
& F_{1}\left(u_{\jmath}\right)=\frac{\left(2-u_{\jmath}^{2}\right)^{2}}{\sqrt{1-u_{\jmath}^{2}}}\left[\frac{1-\exp -\left(\frac{R \gamma_{2} \varphi_{1}\left(u_{\jmath}\right)}{u_{\jmath}}\right)}{\varphi_{1}\left(u_{\jmath}\right)}\right] \\
& \varphi_{1}\left(u_{\jmath}\right)=\left(2-u_{\jmath}^{2}\right)\left(n^{-}-n^{00}\right)+u_{\jmath}^{2} \frac{\left(1-u_{\jmath}^{2}\right)}{\left(2-u_{\jmath}^{2}\right)}\left(n^{0}-n^{00}\right)+\frac{u_{\jmath}^{4}}{\left(2-u_{\jmath}^{2}\right)}\left(n^{+}-n^{00}\right), \\
& F_{3}\left(u_{\jmath}\right)=\sqrt{1-u_{3}^{2}}\left[\frac{1-\exp -\left(\frac{R \gamma_{2} \varphi_{3}\left(u_{\jmath}\right)}{u_{3}}\right)}{\varphi_{3}\left(u_{\jmath}\right)}\right]
\end{aligned}
$$

with

$$
\varphi_{3}\left(u_{\jmath}\right)=\left(1-u_{\jmath}^{2}\right)\left(n^{-}-n^{00}\right)+u_{\jmath}^{2}\left(n^{0}-n^{00}\right)+\left(1-u_{\jmath}^{2}\right)\left(n^{+}-n^{00}\right)
$$

and

$$
F_{5}\left(u_{\jmath}\right)=\frac{1}{\sqrt{1-u_{\jmath}^{2}}}\left[\frac{1-\exp -\left(\frac{R \gamma_{2} \varphi_{5}\left(u_{\jmath}\right)}{u_{\jmath}}\right)}{\varphi_{5}\left(u_{\jmath}\right)}\right]
$$

with

$$
\varphi_{5}\left(u_{\jmath}\right)=\frac{u_{\jmath}^{4}}{\left(2-u_{\jmath}^{2}\right)}\left(n^{-}-n^{00}\right)+u_{\jmath}^{2} \frac{\left(1-u_{\jmath}^{2}\right)}{\left(2-u_{\jmath}^{2}\right)}\left(n^{0}-n^{00}\right)+\left(2-u_{\jmath}^{2}\right)\left(n^{+}-n^{00}\right) .
$$

Similar results can be written for $n^{+}$and $n^{0}$. The detailed calculations are clearly presented in the two Anderson's papers [10]. 
The final necessary parameters are:

i) the initial values of the densities $n^{+}=n^{-}=n^{0}=n / 3$ and $n^{00}=0$

ii) the total laser intensity $I$ per $\mathrm{cm}^{2}$ with $I_{\sigma}=I_{\pi}=I / 2$

iii) the relaxation time $\tau_{\mathrm{r}}$; and

iv) the radius $R$ of the pumping chamber (in our experiment $R=5 \mathrm{~cm}$ ).

The total density $n$ depends on the helium pressure and varies between $10^{9}$ and $10^{11} \mathrm{~cm}^{-3}$.

\section{Results and Discussion}

With the inclusion of the trapping terms the rate equations are no longer linear and, even in their steady-state form, they cannot be solved analytically. Therefore the steady-state limiting values of the densities have been obtained by a numerical integration with respect to time. This numerical integration has been performed by two different codes, run on two different computers. These codes were written independently, using two different numerical integration methods The results were identical within the computer's accuracies. Therefore, any discrepancy between theoretical predictions and experimental data must result from the model, not programming errors.

Figure 3 displays the time evolution of the calculated polarizations, for various metastable initial densities, taking radiation trapping into account. For a density less than $10^{9} \mathrm{~cm}^{-3}$ trapping is negligible and the saturated polarization value is of the order of $80 \%$, while for an initial density larger than $2 \times 10^{11} \mathrm{~cm}^{-3}$ the trapping effect is so strong that this saturated value is reduced to a few percent. Precise values of the upper and lower bounds for the initial density depend on the parameter values used as well as on the approximations made in order to simplify the numerical calculation. The same set of initial metastable density values has been used in a calculation without trapping effect; for any of these densities an asymptotic polarization value close to $85 \%$ is obtained. These results clearly show that, for high metastable initial densities, the trapping effect might account for the low asymptotic polarization value of metastable atoms.

Figure 4 shows the calculated polarization versus the laser intensity with and without trapping: a higher laser intensity will result in a higher polarization and saturation is reached at approximately $13 \mathrm{mWcm}^{-2}$. This theoretical result agrees with the experimental one of Schearer [12]. In the Schearer experiment the polarization is saturated at a $17 \mathrm{mWcm}^{-2}$ intensity while in the Walters experiment the polarization is saturated at $2 \mathrm{~mW} \mathrm{~cm}^{-2}$. If one takes into account the larger absorption cross section in the Rice experiment, this last intensity value would correspond roughly to a $12 \mathrm{~mW} \mathrm{~cm}^{-2}$ intensity in our case. This means that we are interested in laser intensities higher than $15 \mathrm{~mW} \mathrm{~cm}^{-2}$, if possible.

For a total density of $3 \times 10^{10} \mathrm{~cm}^{-3}$, the mean free path of the $1.083 \mu \mathrm{m}$ photons is about $15 \mathrm{~cm}$. Consequently, if the pumping chamber dimension is reduced, the probability of photons escaping from the chamber increases sharply, resulting in lower radiation trapping. This is confirmed by the calculations. Indeed if the radius of the chamber is varied from $7.5 \mathrm{~cm}$ to $2.5 \mathrm{~cm}$ the polarization increases from $68 \%$ to $81 \%$. One concludes that a small dimension of the pumping chamber is the right choice. But, such a reduction leads to shortened diffusion decay lengths and reduces the metastable density and thus the electron currents that can be produced by the source. For instance, in a $1 \mathrm{~cm}$ diameter transport tube the metastables are completely destroyed. So an optimisation procedure must be applied in searching for the proper compromise. 


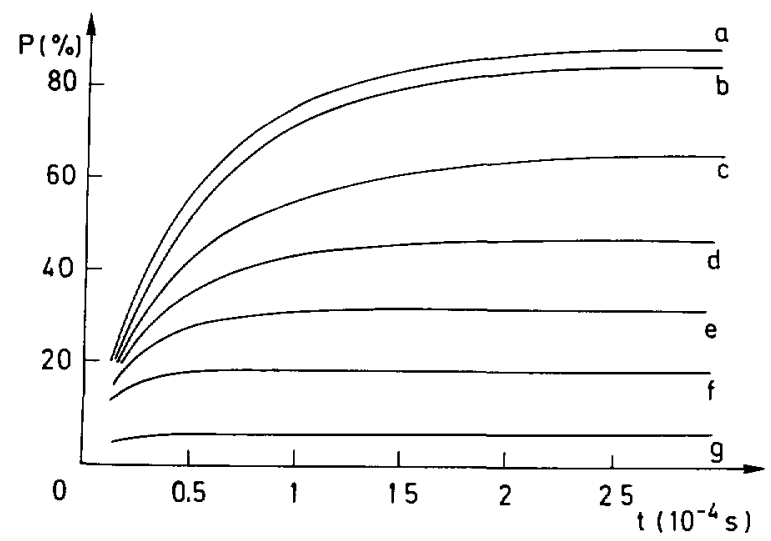

Fig. 3. - Polarization values calculated versus the time $t$ for different metastable densities (a: 0.1, b 10 , c 50 , d 75 , e. 100 , f: $13.0, \mathrm{~g}: 20.0 \mathrm{~m} 10^{10} \mathrm{~cm}^{-3}$ unit). The laser intensity is $7 \mathrm{mWcm}^{-2}$. The radiation trapping is included by contributions (4) to rate equations (1).

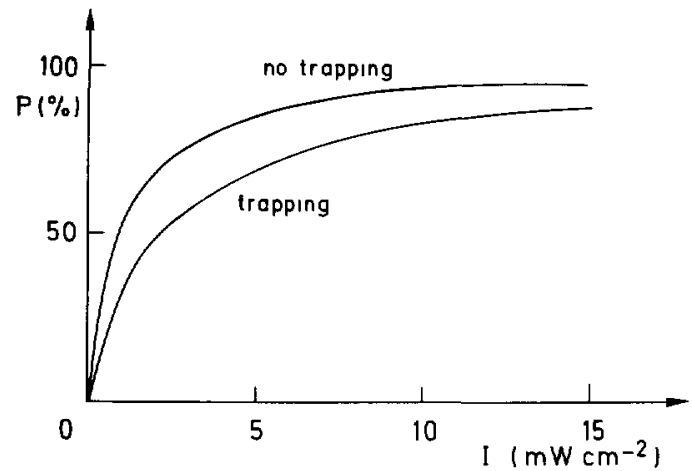

Fig. 4 - Polarization values calculated versus the laser intensity $I$ with and without radiation trapping. The metastable density is $3.0 \times 10^{10} \mathrm{~cm}^{-3}$.

One way to increase the electron current is to increase the metastable density by shortening the transport tube. However this still increases the trapping effect and thus reduces the polarization achievable. Again a compromise must be found.

Figure 5 shows experimental values of the metastable polarization versus the pumping laser power for two different configurations: a) helium pressure $P=0.09 \mathrm{mb}$ and metastable density $n \leq 0.5 \times 10^{10} \mathrm{~cm}^{-3}$; b) helium pressure $P=0.16 \mathrm{mb}$, and metastable density $n \sim 1.4 \times$ $10^{10} \mathrm{~cm}^{-3}$, the other parameters remaining equal (the curves are only a guide for the eye). The difference of shape is due to the trapping effect as is proved by the curves of Figure 4 . This confirms the importance of this effect.

Figure 6 shows the calculated polarizations with the radiation trapping included for laser intensities of 7 and $5 \mathrm{mWcm}^{-2}$ (these values are typical of our laser), as well as experimental metastable and electron polarizations. The experimental values decrease at higher metastable densities following the general trends of the calculated polarizations. Notice however that the experimental polarizations decrease slightly faster than the calculated ones This qualitative agreement confirms the effect of radiation trapping. The metastable experimental values are closer to the theoretical curve than the electron ones. However, one expected approximately the 


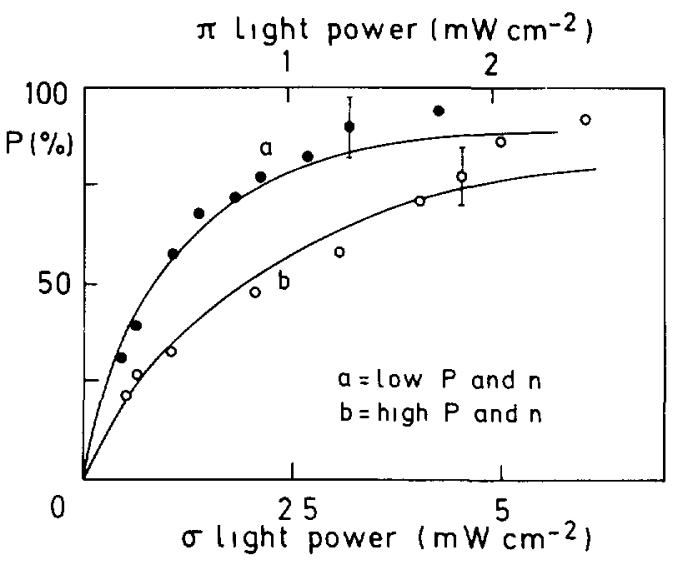

Fig. 5 - Experımental metastable polarization data [6] versus the $\sigma$ light intensity; a) with low helıum pressure and low metastable density; b) with high helium pressure and high metastable density. The curves are only a guide for the eye Radiation trapping explains the lowest polarization in case $\mathrm{b}$.

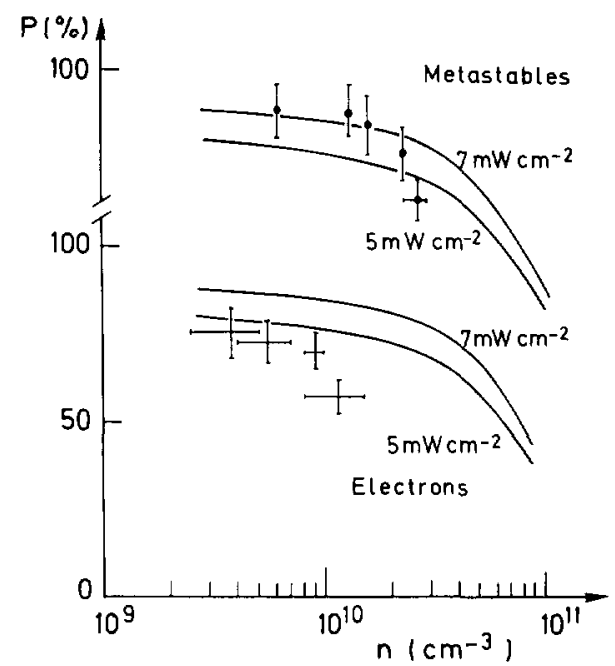

Fig 6 - Polarization values calculated for $I=7$ and $5 \mathrm{mWcm}^{-2}$. The experimental data are the polarization values measured by a probe laser (metastable) and by a Mott polarımeter (electron).

same values for metastable and electron polarization because the spin is well conserved by the chemi-ionization reaction [4]. This large difference indicates that other factors are depolarizing the metastables just before the chemi-ionization and (or) the electrons freed from this reaction before the measurement by the polarimeter. Actually unpolarized electrons are produced by metastables in the singlet state $\left(2^{1} \mathrm{~S}_{0}\right)$. But our measurements indicate for these atoms a relative density less than $5 \%$. Other explanations such as magnetic field gradient effects, depolarizing chemi-ionization concurrent reactions, and elastic collisions of the electrons with the helium gas have been estimated to be negligible by the Rice team [2] and at Orsay, too. Only the ionization of the background gas at the extraction region remains as an explanation of the depolarization. 
It is known [10] that in a cylindrical tube the radial shape of the metastable atom density is well fitted by the function $\cos (\pi r / 2 R)$, where $r$ is the distance to the cylinder axis and $R$ the cylinder radius. We have performed a calculation in which this radial variation of the metastable density is taken into account. This calculation shows a polarization reduction of some ten percent; and thus does not question our conclusions. We are developing a more efficient program in order to take into account not only the metastable density radial variation, but also its longitudinal variation and the nonuniformity of the laser beam as well.

\section{Conclusion}

We have studied the importance of radiation trapping for the Orsay polarized electron source and have shown that this phenomenon explains in a qualitative way the metastable depolarization observed. Recent calculations lead to the same conclusion for the Rice electron source [13]. But at Orsay some other parasitic effects have still to be analyzed to explain the electron polarization degradation. From the present calculations we propose for an improved design of the source:

i) increase the laser intensity;

ii) concentrate the laser light on a smaller pumping volume;

iii) optimize the diameter of the pyrex tube.

\section{Acknowledgments}

Our acknowledgements are due to L. W. Anderson for many concrete comments. G. K. Walters encouraged us to perform this work and it is a pleasure to thank him. We are very grateful to M. Lecuc for many fruitful discussions; N. Sadeghi illuminated different points with patience. We thank him very much. B. Cagnac spent many hours explaining the difficulties of the spatial radiation absorption cross sections. We would like to thank him also. The kindness of G. Rutherford was without limit in sending us information and comments with respect to the Rice University experiment. His cooperation is greatly appreciated. Our thanks are due to M. Campbell and T. Tombrello for reading this paper and to M. Givort for his help in the $\chi^{2}$ procedure calculations. We wish to thank A. Talbot for the perfect typing of the manuscript. This article is dedicated to the memory of L. Schearer who participated in the first stage of the development of the Orsay polarized electron source.

\section{Appendix}

The trapping contributions (Eq. (3)) need the absorption cross sections for the polarized decay radiation: terms $\sigma_{j}^{\imath}$ where $\imath$ means the $\Delta m$ for emission and $\jmath$ the $\Delta m$ for absorption. The quantities are specific for the helium $2^{3} \mathrm{~S}_{1}-2^{3} \mathrm{P}_{0}$ transition. Following reference [10]:

with

$$
\begin{aligned}
\sigma_{0}^{0}(\theta) & =2 \sigma \sin ^{2} \theta \\
\sigma_{+1}^{0}(\theta) & =\sigma_{-1}^{0}(\theta)=\sigma \cos ^{2} \theta \\
\sigma_{+1}^{+1}(\theta) & =\sigma_{-1}^{-1}(\theta)=\sigma \sin ^{4} \theta /\left(1+\cos ^{2} \theta\right) \\
\sigma_{-1}^{+1}(\theta) & =\sigma_{+1}^{-1}(\theta)=\sigma\left(1+\cos ^{2} \theta\right) \\
\sigma_{0}^{+1}(\theta) & =\sigma_{0}^{-1}(\theta)=2 \sigma \sin ^{2} \theta /\left(1+\cos ^{2} \theta\right) \\
\sigma & =\frac{\lambda^{2}}{16 \pi \tau} F\left(v_{0}\right)
\end{aligned}
$$




\section{References}

[1] Arianer J., Aminoff C. G., Brissaud I., Proceedings of the Workshop on "Future of Nuclear Physics", Orsay (July 1990).

[2] Rutherford G. H., Ratliff J. M., Lynn J. G., Dunning F. B, Walters G K., Rev Scz. Instrum. 61 (1990) 1460 and references therein.

[3] Arianer J., Brissaud I., Humblot H., Zerhouni W., Nucl. Instr Meth. A337 (1993) 1.

[4] Keliher P. J., Ph.D. Thesis (1975), Rice University, unpublished,

Walters G R., private communication.

[5] Ferguson E. E. et al., Advances in Atomic and Molecular Physics, vol. 5 (Academic Press, New York, 1969).

[6] Schearer L., Arianer J, Brissaud I., Humblot H., Zerhouni W, Nucl Instr. Meth. A344 (1994) 315.

[7] Mitchell A C G., Zemanskı M. W., Resonance radiation and excited atoms (Cambridge University Press, London, 1961)

[8] Corney A., Atomic and Laser Spectroscopy (Clarendon Press, Oxford, 1979).

[9] Phelps A. V., Phys. Rev 99 (1955) 1307,

Phelps A. V., Molnar J P., Phys Rev. 89 (1953) 1202

[10] Tupa D, Anderson, L. W., Huber D. L., Lawler J. E., Phys. Rev. A 33 (1986) 1045;

Tupa D., Anderson L. W., Phys. Rev. A 36 (1987) 2142

[11] Abramowitz M, Stegun I. A , Handbook of Mathematıcal Functions with Formulas, Graphs, and Mathematical Tables, chap 7 (Dover, New York, 1970).

[12] Schearer L. D., Padetha Tin, Phys. Rev. A 42 (1990) 4028.

[13] Brissaud I, in preparation. 